\title{
A review of applying second-generation wavelets for noise removal from remote sensing data.
}

\begin{abstract}
The processing of remotely sensed data includes compression, noise reduction, classification, feature extraction, change detection and any improvement associated with the problems at hand. In the literature, wavelet methods have been widely used for analysing remote sensing images and signals. The second-generation of wavelets, which is designed based on a method called the lifting scheme, is almost a new version of wavelets, and its application in the remote sensing field is fresh. Although first-generation wavelets have been proven to offer effective techniques for processing remotely sensed data, second-generation wavelets are more efficient in some respects, as will be discussed later. The aim of this review paper is to examine all existing studies in the literature related to applying second-generation wavelets for denoising remote sensing data. However, to make a better understanding of the application of wavelet-based denoising methods for remote sensing data, some studies that apply first-generation wavelets are also presented. In the part of hyperspectral data, there is a focus on noise removal from vegetation spectrum.
\end{abstract}

Keyword: Remote sensing; Wavelets; Denoising 